\title{
Many-spin quantum dynamics during cross polarization in 8CB
}

\author{
Ana K. Chattah, Gonzalo A. Álvarez, Patricia R. Levstein, ${ }^{\text {a) }}$ Fernando M. Cucchietti, \\ and Horacio M. Pastawski \\ Facultad de Matemática, Astronomía y Física, Universidad Nacional de Córdoba, Ciudad Universitaria, \\ 5000 Córdoba, Argentina
}

\author{
Jésus Raya and Jérôme Hirschinger \\ Laboratoire de RMN de la Matière Condensée, Institut Le bel, Université Louis Pasteur, 67000, \\ Strasbourg, France
}

(Received 12 May 2003; accepted 23 July 2003)

\begin{abstract}
We analyze theoretically and experimentally the quantum dynamics of a three-spin- $1 / 2$ system during cross polarization (CP). Our analysis takes into account a Hamiltonian behavior for a carbon ${ }^{13} \mathrm{C}$ coupled to two protons ${ }^{1} \mathrm{H}$ while the coupling to a spin bath is treated in the fast fluctuation approximation. This model is applied to the methylene and biphenyl groups of the smectic and nematic phases of the liquid crystal 4 - $n$-octyl- 4 ' -cyanobiphenyl (8CB). Experimental data from standard $\mathrm{CP}$, combined with our theoretical results, allow us to separate the homonuclear ${ }^{1} \mathrm{H}-{ }^{1} \mathrm{H}$ and heteronuclear ${ }^{1} \mathrm{H}-{ }^{13} \mathrm{C}$ residual dipolar couplings. These values are in good agreement with those obtained by using a combination of $\mathrm{CP}$ under Lee-Goldburg conditions and standard $\mathrm{CP}$ data. A well differentiated relaxation behavior among the two phases seems to indicate that while the extreme narrowing approximation is appropriate for the nematic phase, the description of the smectic phase requires consideration of the slow-motion limit. (C) 2003 American Institute of Physics. [DOI: 10.1063/1.1609394]
\end{abstract}

\section{INTRODUCTION}

The exact quantum dynamics of few-spin systems has regained interest during the last years. This fact responds to challenging applications requiring very fine knowledge of the spin interactions, such as molecular characterization, spin control in nanodevices, ${ }^{1}$ and quantum computation ${ }^{2}$ as well as to more fundamental reasons. On this side, the limitations of simple thermodynamical arguments based on the spin temperature hypothesis have become evident. Ingenious nuclear magnetic resonance (NMR) experiments have demonstrated the time reversibility of the dipolar (many-spin) evolution, ${ }^{3-6}$ leading to a revision of the concept of spin diffusion. ${ }^{7-9}$ Then, by selecting appropriate systems and pulse sequences, one can investigate the sources of quantum decoherence, ${ }^{10}$ ergodicity, ${ }^{7,9}$ and quasiequilibrium. ${ }^{11}$

On the other side, the spin dynamics observed by NMR has proved to be very suitable for characterizing molecular structures and dynamics. ${ }^{12}$ Experimental observations together with simple analytical solutions for few-spin dynamics can provide detailed information on the intramolecular and intermolecular interactions. ${ }^{7,13}$ This is particularly important for the characterization of complex fluids in their native state, where one uses cross polarization $(\mathrm{CP})$ dynamics to evaluate order parameters. ${ }^{14}$ However, the reliability of these and other structural and dynamical parameters depends on the accuracy of the spin dynamics description to which the experimental data are fitted.

In this paper, we study how the Hartmann-Hahn cross-

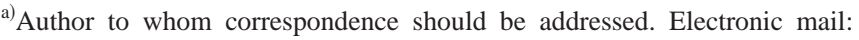
patricia@famaf.unc.edu.ar
}

polarization dynamics can be exploited to measure ${ }^{1} \mathrm{H}-{ }^{13} \mathrm{C}$ and ${ }^{1} \mathrm{H}-{ }^{1} \mathrm{H}$ effective dipolar interactions in the nematic and smectic phases of the liquid crystal 4- $n$-octyl-4' -cyanobiphenyl (8CB). Most of the previous works where transient oscillations were observed during $\mathrm{CP}$ were analyzed in terms of a single ${ }^{1} \mathrm{H}-{ }^{13} \mathrm{C}$ interaction incorporating the coupling with other protons as a thermal bath or reservoir in a phenomenological way. ${ }^{13}$ However, as occurs in $8 \mathrm{CB}$, many liquid crystals have alkyl chains and aromatic groups in their structure, where the carbon is coupled to more than one proton and the carbon-proton and proton-proton dipolar interactions are of the same order of magnitude. This led us to consider a set of three strongly dipolar coupled spins $1 / 2$ as the main system, which interacts with the protons of the bath. We apply the three-spin model together with structural information, which yields the relative signs of the heteronuclear couplings in oriented $8 \mathrm{CB}$, to obtain the ${ }^{1} \mathrm{H}-{ }^{13} \mathrm{C}$ and ${ }^{1} \mathrm{H}-{ }^{1} \mathrm{H}$ effective interactions. Our calculations provide values for the homonuclear dipolar interactions, which are ignored in the single ${ }^{1} \mathrm{H}-{ }^{13} \mathrm{C}$ model, improving the evaluation of the heteronuclear interactions in more than $10 \%$. In order to test our solution for the evolution of the three-spin system, we compare the values of the ${ }^{1} \mathrm{H}-{ }^{13} \mathrm{C}$ couplings obtained by two procedures. One fits the data from a standard $\mathrm{CP}$ experiment to the calculated dynamics. The other evaluates ${ }^{1} \mathrm{H}-{ }^{13} \mathrm{C}$ couplings directly from a CP under Lee-Goldburg conditions-i.e., when the dipolar proton-proton interactions have been canceled out. The advantages and disadvantages of each procedure are discussed.

An interesting aspect we observed during the $\mathrm{CP}$ dynamics in $8 \mathrm{CB}$ is that the rate of attenuation of the oscillations (representing the coherences) is much faster than that of the 


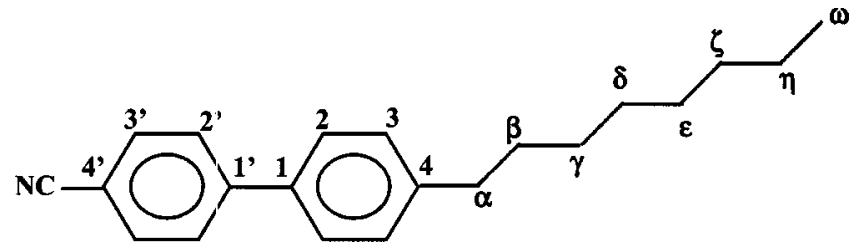

FIG. 1. Chemical structure of 4- $n$-octyl-4' -cyanobiphenyl (8CB) showing the labels used in the ${ }^{13} \mathrm{C}-\mathrm{NMR}$ spectra.

polarization transfer from the bath. The factor is several times larger than the one calculated assuming isotropic ${ }^{13}$ interactions with the reservoir. This anisotropic behavior exceeds that observed in solid molecular crystals. ${ }^{6,15}$ In order to analyze these observations, we introduce an interaction Hamiltonian that can take into account different couplings with the spin bath (dipolar, isotropic, etc.). In particular, we find that the dipolar interaction is enough to explain the anisotropy observed in molecular crystals. Comparison of the spin dynamics in the nematic and smectic phases of $8 \mathrm{CB}$ indicate different relaxation behaviors, requiring different approaches with regard to fluctuations of the spin bath.

\section{EXPERIMENTAL METHODS}

Nuclear magnetic resonance experiments were carried out in 4-n-octyl-4' - cyanobiphenyl, also called $8 \mathrm{CB}$ (see Fig. 1), obtained from Sigma-Aldrich Inc. and used without further purification. This system presents the mesophases smectic $\mathrm{A}(\mathrm{SA})$ and nematic $(\mathrm{N})$, with transition temperatures at $294.5 \mathrm{~K}(\mathrm{~K}-\mathrm{SA}), 306.5 \mathrm{~K}(\mathrm{SA}-\mathrm{N})$, and $313.5 \mathrm{~K}(\mathrm{~N}-\mathrm{I})$.

${ }^{1} \mathrm{H}-{ }^{13} \mathrm{C}$ cross polarization measurements as a function of contact time $t_{c}$ were performed in the smectic and nematic mesophases. In all the cases, the CP sequence was performed in static conditions and combined with the sequence SPINAL-64 to perform an efficient proton decoupling during acquisition without appreciable heating of the sample. ${ }^{16}$

In the smectic phase, standard CP experiments were performed at $300 \mathrm{~K}$ in a Bruker MSL-300. The acquisition time was $92 \mathrm{~ms}$, with $60 \mathrm{~ms}$ for decoupling and a recycling time of $15 \mathrm{~s}$. The Hartmann-Hahn (HH) condition was set with an rf field amplitude for carbons corresponding to $\omega_{1 S} / 2 \pi$ $=67.7 \mathrm{kHz}$. During the experiment the contact time was varied in the range $2 \mu \mathrm{s}<t_{c}<5 \mathrm{~ms}$.

In the nematic phase at $311.5 \mathrm{~K}$ two types of $\mathrm{CP}$ experiments were performed in a Bruker AVANCE DSX-500. The first was a standard $\mathrm{CP}$ with protons on resonance. The second was a $\mathrm{CP}$ experiment with irradiation for protons in the Lee-Goldburg (LG) condition: i.e., the off resonance for protons was set to have an effective field at the magic angle with static field $H_{0}$. The acquisition and decoupling times were $74 \mathrm{~ms}$. In the standard $\mathrm{CP}$, the $\mathrm{HH}$ condition was set with $\omega_{1 S} / 2 \pi=60.3 \mathrm{kHz}$ while in the Lee-Goldburg condition $\omega_{1 S} / 2 \pi=74 \mathrm{kHz}$. In both sets of experiments the $\mathrm{HH}$ condition was optimized for $C(\gamma)$ (see Figs. 1 and 2), and the contact time $t_{c}$ varied up to $2 \mathrm{~ms}$.

In all these experiments the temperatures were calibrated using the $\mathrm{N}-\mathrm{I}$ temperature transition. Also a ${ }^{13} \mathrm{C}-\mathrm{NMR}$ spec- trum of $8 \mathrm{CB}$ in the isotropic phase (at $320 \mathrm{~K}$ ) was taken as reference using a single pulse sequence with ${ }^{1} \mathrm{H}$ decoupling (direct ${ }^{13} \mathrm{C}$ polarization).

\section{SPIN DYNAMICS CALCULATIONS}

We will consider the evolution of a system of three spins $1 / 2$ coupled through the magnetic dipolar interaction during the contact time in a cross-polarization experiment. The system is constituted by one spin $S$ and two spins $I$ representing a carbon-13 and two protons, respectively, under the presence of a static magnetic field $H_{0}$ in the $z$ direction and radio-frequency (rf) magnetic fields $H_{1 I}$ and $H_{1 S}$ in the $x$ direction. The Hamiltonian includes the dipolar interactions truncated with respect to the Zeeman field $H_{0}$. In the doublerotating frame it can be written as

$$
\begin{aligned}
\frac{\mathcal{H}}{\hbar}= & \Delta \omega_{I} I^{z}-\omega_{11} I^{x}+\Delta \omega_{S} S^{z}-\omega_{1 S} S^{x}+2 \sum_{k=1,2} b_{k} S^{z} I_{k}^{z} \\
& +d\left(2 I_{1}^{z} I_{2}^{z}-I_{1}^{x} I_{2}^{x}-I_{1}^{y} I_{2}^{y}\right),
\end{aligned}
$$

where $I^{u}=I_{1}^{u}+I_{2}^{u}$ with $u=x, y, z$. The resonance offsets are $\Delta \omega_{I}=\omega_{0 I}-\omega_{I}$ and $\Delta \omega_{S}=\omega_{0 S}-\omega_{S}$. The rf fields give $\omega_{1 I}$ $=\gamma_{I} H_{1 I}$ and $\omega_{1 S}=\gamma_{S} H_{1 S}$ where $\gamma_{I}$ and $\gamma_{S}$ are the gyromagnetic factors of the $I$ and $S$ spins. The constants

$$
b_{k}=-\frac{\mu_{0} \gamma_{I} \gamma_{S} \hbar}{4 \pi} \frac{1}{2}\left\langle\frac{\left(3 \cos ^{2}\left(\theta_{S k}\right)-1\right)}{r_{S k}^{3}}\right\rangle, \quad k=1,2,
$$

and

$$
d=-\frac{\mu_{0} \gamma_{I}^{2} \hbar}{4 \pi} \frac{1}{2}\left\langle\frac{\left(3 \cos ^{2}\left(\theta_{12}\right)-1\right)}{r_{12}^{3}}\right\rangle
$$

are the heteronuclear and homonuclear effective dipolar couplings, respectively. Here, $r_{i j}$ is the spin-spin distance and $\theta_{i j}$ is the angle between the internuclear vector and external field. The angular brackets indicate that the dipolar couplings in liquid crystals are averaged over both molecular tumbling and any internal bond rotation. Because of the special geometry of the oriented $n \mathrm{CB}$ liquid crystals, we will consider two different cases where the dipolar constants are related by $b_{1}=b_{2}=b$ and $b_{1}=-b_{2}=b$.

For a standard CP experiment, one can neglect the resonance offsets. Considering that $\left|\omega_{1 I}+\omega_{1 S}\right| \gg\left|b_{k}\right|,|d|$, the truncated Hamiltonian can be written as

$$
\begin{aligned}
\frac{\mathcal{H}}{\hbar}= & \frac{1}{2}\left[\Sigma\left(I^{x}+S^{x}\right)+\Delta\left(I^{x}-S^{x}\right)+2 \sum_{k=1,2} b_{k}\left(S^{z} I_{k}^{z}+S^{y} I_{k}^{y}\right)\right. \\
& \left.-d\left(2 I_{1}^{x} I_{2}^{x}-I_{1}^{z} I_{2}^{z}-I_{1}^{y} I_{2}^{y}\right)\right]
\end{aligned}
$$

with $\Sigma=-\left(\omega_{1 I}+\omega_{1 S}\right)$ and $\Delta=\left(\omega_{1 S}-\omega_{1 I}\right)$. In Eq. (4) nonsecular elements of the dipolar interaction with respect to the $\Sigma\left(I^{x}+S^{x}\right)$ term have been neglected. This allows us to write the matrix representation of the Hamiltonian in a simple block structure using the basis $\left\{\left|M_{I}, M_{S}\right\rangle\right\}$, with $M_{I}=M_{1}$ $+M_{2}$ and $M_{S}$ denoting the spin projections of the $I$ and $S$ systems in the direction of their respective rf fields. Each block is characterized by the total spin projection $M=M_{I}$ $+M_{S}$; i.e., nonzero matrix elements exist only between 
states with the same magnetic quantum numbers $M$. Thus, the heteronuclear dipolar Hamiltonian has nondiagonal terms different from zero generating transitions between spin states $\left\{\left|M_{I}, M_{S}\right\rangle\right\}$ and $\left\{\left|M_{I} \pm 1, M_{S} \mp 1\right\rangle\right\}$. The eigenstates of this Hamiltonian can be denoted in the form $\left|M, n_{M}\right\rangle$, with $n_{M}$ $=1, \ldots, g_{M}$, where $g_{M}$ is the degeneracy of $M\left(n_{ \pm 3 / 2}=1\right.$ and $\left.n_{ \pm 1 / 2}=1,2,3\right)$. It is very interesting to note that in each space of $M= \pm 1 / 2$ there are only two of the three eigenstates that are involved in the dipolar transitions that give rise to oscillations. This is a consequence of the symmetry of the system: i.e., the flip-flop can occur only between the carbon and only one combination of the proton states, either the symmetric or antisymmetric depending on the relative signs of the heteronuclear couplings $\left(b_{1}=b_{2}\right.$ or $\left.b_{1}=-b_{2}\right)$.

The Liouville-von Neumann equation for the density matrix of the system is

$$
\frac{d}{d t} \sigma(t)=-\frac{\mathrm{i}}{\hbar}[\mathcal{H}, \sigma(t)],
$$

where the initial density operator $\sigma(0)$ considers the situation after the $\pi / 2$ pulse in the $I$ system. Under the hightemperature approximation $\sigma(0)=\left(\mathbf{1}+\beta \hbar \omega_{0 I} I^{x}\right) / \operatorname{Tr}\{\mathbf{1}\}$, with $\beta=1 / k_{B} T$.

In the simplest case, where the Hartmann-Hahn condition is exactly fulfilled $(\Delta=0)$, the exact solution for the evolution of the observed magnetization $M_{S^{x}}(t)$ is

$$
M_{S^{x}}(t)=\operatorname{Tr}\left\{S^{x} \sigma(t)\right\}=M_{0} f \frac{1-\cos \left(\omega_{c p} t\right)}{2},
$$

where

$$
\omega_{c p}=\sqrt{\left(\frac{\kappa}{4}\right)^{2} d^{2}+2 b^{2}} \text { and } f=2 b^{2} / \omega_{c p}^{2},
$$

with

$$
\kappa= \begin{cases}1 & \text { if } b_{1}=-b_{2}=b, \\ 3 & \text { if } b_{1}=b_{2}=b .\end{cases}
$$

The frequency $\omega_{c p}$ of the polarization transfer corresponds to the transitions between the eigenstates mentioned above. Now, it is clear that the symmetry of the system manifests directly in the frequency, where the difference between the two situations is represented through the $\kappa$ parameter. The constant $M_{0}=\beta \hbar \omega_{0 I} / 4$ corresponds to the initial magnetization of one $I$ spin. Equation (6) shows that the magnetization of $S$ is attenuated by the factor $f$, and it takes its maximum value $M_{0}$ when $d=0$. The fact that the homonuclear interaction decreases the transferred magnetization was already noticed in a previous work. ${ }^{7}$ We can see that the constant term in Eq. (6) is proportional to the differences in populations between the relevant eigenstates of the system, while the time-dependent term corresponds to the coherences representing the transitions from $\left\{\left|M_{I}, M_{S}\right\rangle\right\}$ to $\left\{\mid M_{I} \pm 1, M_{S}\right.$ $\mp 1\rangle$ \}

The interaction of other spins (the bath) with the threespin system is included by extending the model proposed by Müller et al. ${ }^{13}$ The model assumes that the dipolar interactions of the $S$ spin with the $I$ spins are neglected except for the coupling to $I_{1}$ and $I_{2}$. The interaction of these particular spins with the bath or infinite reservoir of $I$ spins is considered in a phenomenological way. All kind of spin-lattice relaxations are neglected. The interaction Hamiltonian can be represented by

$$
\mathcal{H}_{\mathrm{int}}=\sum_{k=1,2}\left[\alpha F_{k}^{x} I_{k}^{x}-\left(F_{k}^{y} I_{k}^{y}+F_{k}^{z} I_{k}^{z}\right)\right]
$$

with $F_{k}^{u}=[-1 / 2] \Sigma_{l} d_{k l} I_{l \mathrm{~B}}^{u}$ where the subscript B corresponds to the bath. For a truncated dipolar Hamiltonian, $\alpha=2$, and the exact factor $[-1 / 2]$ comes from the truncation with respect to the rf field. For an isotropic interaction (Heisenberg) $\alpha=-1$ and the factor [ $-1 / 2]$ does not appear. In quantum mechanical relaxation theory, the terms $F_{k}^{u}$ are bath operators. In the semiclassical theory, by tracing on the bath variables, they are treated as temporal functions $F_{k}^{u}(t)$ representing classical random processes. This semiclassical theory is consistent with a quantum treatment in the infinitetemperature approximation. Then, the random interaction Hamiltonian is written as

$$
\mathcal{H}_{\text {int }}(t)=\sum_{k=1,2}\left\{\alpha F_{k}^{x}(t) I_{k}^{x}-\left[F_{k}^{y}(t) I_{k}^{y}+F_{k}^{z}(t) I_{k}^{z}\right]\right\}
$$

This interaction takes into account not only the spin dynamics of the bath but the effect of other degrees of freedom (rotations, translations, etc.). The time averages of these random processes at infinite temperature satisfy $\overline{F_{k}^{u}(t)}=0$, with correlation functions $g_{k}^{(u, v)}(\tau)=\overline{F_{k}^{u}(t) F_{k}^{v *}(t+\tau)}$. Within the second-order approximation, the dynamics of the reduced density operator is ${ }^{17-19}$

$$
\frac{d}{d t} \sigma(t)=-\frac{\mathrm{i}}{\hbar}[\mathcal{H}, \sigma(t)]-\hat{\Gamma}\left\{\sigma(t)-\sigma_{0}\right\} .
$$

The relaxation superoperator $\hat{\hat{\Gamma}}$ is generated by $\mathcal{H}_{\text {int }}(t)$ and accounts for dissipative interactions between the reduced spin system and the bath. It drives the density operator towards its equilibrium value $\sigma_{0}$. In the semiclassical theory, $\sigma_{0}$ gives the information of the high (but not infinite) final temperature of the system.

We assume that the correlation times of the fluctuations are extremely short compared with all the relevant transition rates between eigenstates of the Hamiltonian-i.e., frequencies of the order of $\Sigma / 2$ and $\omega_{c p}$. In this extreme narrowing regime, we obtain

$$
\hat{\Gamma}\{\sigma\}=\frac{1}{2} \sum_{k} \sum_{u, v} \alpha_{u, v} J_{k}^{(u, v)}(0)\left[I_{k}^{u},\left[I_{k}^{v}, \sigma\right]\right],
$$

where $J_{k}^{(u, v)}(\omega)=\int_{-\infty}^{\infty} d \tau g_{k}^{(u, v)}(\tau) \exp \{-\mathrm{i} \omega \tau\}$ is the spectral density and $\alpha_{u, v}=\left(\alpha \delta_{u, x}-\delta_{u, y}-\delta_{u, z}\right)\left(\alpha \delta_{v, x}-\delta_{v, y}-\delta_{v, z}\right)$. The spatial directions are statistically independent-i.e., $g_{k}^{(u, v)}(\tau)=0$ if $u \neq v$ : then, only terms with $J_{k}^{(u, u)}(0)$ $\equiv 2 R_{k u}$ survive in the superoperator $\hat{\hat{\Gamma}}$. Notice that the axial symmetry of $\mathcal{H}$ around the $x$ axis leaves as observable the averaged value $R_{k \perp}=\left(R_{k y}+R_{k z}\right) / 2$. An extra simplification arises from the symmetry $b_{1}= \pm b_{2}$, leading to $R_{u}=\left(R_{1 u}\right.$ $\left.+R_{2 u}\right) / 2$. Then, we obtain 


$$
\begin{aligned}
\hat{\Gamma}\{\sigma\}= & \sum_{k=1,2} \alpha^{2} R_{\|}\left[I_{k}^{x},\left[I_{k}^{x}, \sigma\right]\right] \\
& +R_{\perp}\left(\left[I_{k}^{y},\left[I_{k}^{y}, \sigma\right]\right]+\left[I_{k}^{z},\left[I_{k}^{z}, \sigma\right]\right]\right) .
\end{aligned}
$$

Although we could absorb the constant $\alpha^{2}$ in $R_{\|}$, we will keep it to emphasize the different sources of the anisotropy in Eq. (13). The most usual approximation is to consider $R_{x}=R_{y}=R_{z}$ (identical correlations in all the spatial directions) and $\alpha=-1$ (isotropic interaction Hamiltonian). ${ }^{13} \mathrm{~A}$ better approximation considers a dipolar interaction Hamiltonian-i.e., $\alpha=2$. This is in excellent agreement with previous works in polycrystalline samples where fittings to phenomenological equations have been performed. ${ }^{6,20}$ In particular, in the case of isotactic polypropylene, ${ }^{20}$ a fitting where $R_{d p}$ corresponds to $\alpha^{2} R_{\|}$and $R_{d f}=R_{\perp}$ gives $R_{d p} / R_{d f} \sim 4$.

Following the standard formalism, ${ }^{19}$ we write the superoperator $\hat{\hat{\Gamma}}$ using the basis of eigenstates of the Hamiltonian (4). After neglecting the rapidly oscillating nonsecular terms with respect to the Hamiltonian-i.e., $R_{\|}, R_{\perp} \ll|b|,|d|-\mathrm{a}$ block structure results. The first block couples the populations and off-diagonal elements with $\Delta M=0$, zero quantum transitions (ZQT), of the density matrix. Each of the following blocks couples one order $\Delta M \geqslant 1$ of off-diagonal elements of the density matrix among themselves. Because the Hamiltonian (4) does not have degenerate eigenenergies, all nondiagonal terms coupling the population block with the ZQT block are nonsecular and can be neglected. As the initial condition $\sigma(0)$ does not contain coherences with $\Delta M$ $\geqslant 1$, we only need to study the evolution of the density operator into a Liouville space restricted to populations and ZQT. When there are no degenerate transitions, the secular ZQT block is diagonal. However, in our case there are degenerate transitions between eigenstates within the sets with $M= \pm 1 / 2$. Thus, some nondiagonal terms in the ZQT block cannot be neglected.

In the final state, the $I_{2} S$ system reaches the temperature of the $I$ spins reservoir:

$$
\sigma_{0}=\frac{\mathbf{1}+\beta \hbar \omega_{0 I}\left(I^{x}+S^{x}\right)}{\operatorname{Tr}\{\mathbf{1}\}} .
$$

It is easily seen that $\sigma_{0}$ commutes with $\mathcal{H}$, not containing coherences with $\Delta M \geqslant 1$.

Under the considered approximations, we solve Eq. (11) for the cases relevant to $8 \mathrm{CB}$.

Isotropic case solution: considering $R_{\|}=R_{\perp}=R$ and $\alpha$ $=-1$, the time evolution of the $S$ magnetization results

$$
\begin{aligned}
M_{S^{x}}(t)= & M_{0}\left[1-A_{+} e^{-R_{+} t}-A_{-} e^{-R_{-} t}\right. \\
& \left.-\frac{1}{2} f \cos \left(\omega_{c p} t\right) e^{-R_{\mathrm{c}} t}\right],
\end{aligned}
$$

where $R_{ \pm}=\chi_{ \pm} R, R_{\mathrm{c}}=\chi_{\mathrm{c}} R$, with

$$
\begin{aligned}
& \chi_{ \pm}=1+\frac{5}{4} f \pm \sqrt{\left(\frac{5}{4} f-1\right)^{2}+f}, \\
& \chi_{\mathrm{c}}=3-\frac{5}{4} f,
\end{aligned}
$$

and

$$
A_{ \pm}=\frac{1}{2}\left\{\left(1-\frac{f}{2}\right) \pm \frac{\left[\frac{5}{4} f\left(1-\frac{f}{2}\right)-1\right]}{\sqrt{\left(\frac{5}{4} f-1\right)^{2}+f}}\right\} .
$$

Notice that $\chi_{ \pm}, \chi_{\mathrm{c}} \geqslant 0$. Using the initial condition $M_{S^{x}}(0)$ $=0$, it is easy to see that the positive constants $A_{+}, A_{-}$ satisfy $1-A_{+}-A_{-}-\frac{1}{2} f=0$. In general $A_{+} \ll A_{-}$and $R_{+}$ $>R_{-}$, so the first exponential term can be neglected. This approximate solution is excellent for $f \ll 1$, but even in the worst case $(f \sim 1)$, it differs about $7 \%$ from the exact solution.

The first maximum in the magnetization $M_{S^{x}}(t)$ is approximately $f M_{0}$, and the oscillation has frequency $\omega_{c p}$ and amplitude $(f / 2) e^{-R_{\mathrm{c}} t}$ representing the attenuation of the coherences of the $I_{2} S$ system mounted over nonoscillatory terms. These terms take into account the effect of the bath. They do not only transfer magnetization, but they break coherences and lead to a quasiequilibrium characterized by $\sigma^{q e}=\exp \left\{-\mathcal{H} /\left(k_{B} T_{q e}\right)\right\} / \operatorname{Tr}\left\{\exp \left[-\mathcal{H} /\left(k_{B} T_{q e}\right)\right]\right\} \quad$ with $T_{q e}$ $=3 / 2\left(\omega_{1 I} / \omega_{0 I}\right) T$, the temperature of the three-spin system.

In the particular case where $f=1$, i.e., no $I_{1}-I_{2}$ interaction, $A_{ \pm}=\frac{1}{4}(1 \mp 3 / \sqrt{17}), R_{ \pm}=\frac{1}{4}(9 \pm \sqrt{17}) R, R_{\mathrm{c}}=7 / 4 R$, and $\omega_{c p}=\sqrt{2} b$. The frequency given in Ref. 14 is valid only under this condition. But even in this case, our results show that the equation obtained by Müller et al. for the IS system cannot be directly applied to the $I_{2} S$. In this last case, the attenuation of the oscillations and the transfer of polarization from the bath are slightly faster than those of the $I S$ system.

Anisotropic case solution: considering $\alpha^{2} R_{\|} \neq R_{\perp}$ we obtain

$$
\begin{aligned}
M_{S^{x}}(t)= & M_{0}\left[1-A_{1} e^{-R_{1} t}-A_{2} e^{-R_{2} t}-A_{3} e^{-R_{3} t}\right. \\
& \left.-\frac{1}{2} f \cos \left(\omega_{c p} t\right) e^{-R_{c} t}\right],
\end{aligned}
$$

where $A_{i}=A_{i}\left(f, R_{\|} / R_{\perp}\right), \quad R_{i}=R_{i}\left(f, R_{\|}, R_{\perp}\right)$ with $i=1,2,3$ too long to be included here, and

$$
R_{\mathrm{c}}=(2-f) R_{\perp}+\left(1-\frac{1}{4} f\right) \alpha^{2} R_{\|} .
$$

The transfer of polarization from the bath to the system depends on the nonoscillatory terms of Eq. (19). In the case $\alpha^{2} R_{\|} / R_{\perp} \geqslant 1$, at long times $\left(R_{\mathrm{c}} t \gg 1\right)$, only one of the three exponential terms contributes. In this regime, the transfer is essentially given by $R_{\perp}$, although there is a slight dependence on $R_{\|}$. This differs from the $I S$ behavior where the polarization transfer from the bath depends exclusively on $R_{\perp} \cdot{ }^{15}$ This is a consequence of the fact that in the $I S$ system the quasiequilibrium $S^{x}$ polarization $(1 / 2) M_{0}$ coincides with the time-averaged value of the isolated system. As $R_{\perp}$ is associated to the flip-flop term in the interaction Hamiltonian (9), its role in transferring polarization can be easily interpreted. The effect of $R_{\|}$is more subtle: it can be associated with a process where the environment observes the system breaking its coherences. This process involves the operator $F^{x} I^{x}$ in $\mathcal{H}_{\text {int }}$. As $R_{\|}$is always multiplied by $\alpha^{2}$, it is easy to see that the dipolar interaction is more effective than the isotropic and this, in turn, more effective than the $X Y$ (planar, $\alpha=0$ ) interaction to produce decoherence. 


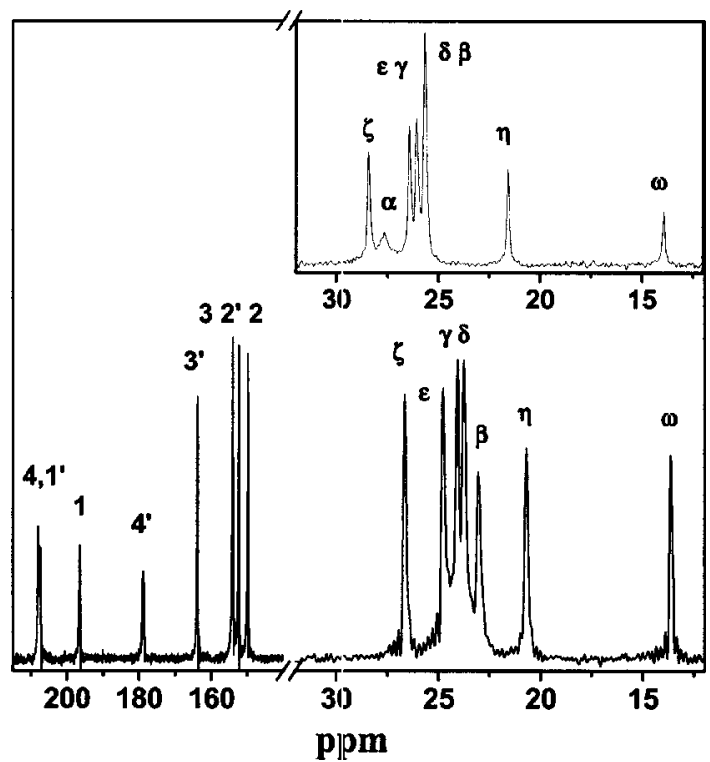

FIG. $2 .{ }^{13} \mathrm{C}$-NMR spectra of $8 \mathrm{CB}$ in the smectic mesophase at $300 \mathrm{~K}$. The inset displays the aliphatic part of the spectrum corresponding to $311.5 \mathrm{~K}$ (nematics). The aromatic lines keep their relative positions up to the nematic-isotropic transition temperatures.

In the limit $R_{\|} \gg R_{\perp}$ (highly anisotropic case), it is possible to distinguish two time regimes: one in which the system decoheres and reaches a quasiequilibrium, $\left[\mathcal{H}, \sigma^{q e}\right]$ $=0,{ }^{11}$ characterized by $R_{\|}$, and other in which polarization transfer from the bath is completed with a rate $R_{\perp}$. In this situation, it is possible to see that $R_{\mathrm{c}}$ is proportional to $R_{\|}$, as it occurs with the $R_{i}$ corresponding to the fastest exponential terms. The dependence of the nonoscillatory terms on $R_{\|}$ observed in the $I_{2} S$ system can be assigned to the fact that the quasiequilibrium carbon polarization $(2 / 3) M_{0}$ does not coincide with the time-averaged value $(f / 2) M_{0}$ of the isolated three-spin system [Eq. (6)].

Equations (15) and (19) will be used to fit the experimental data in order to extract the relevant parameters of our system.

\section{RESULTS AND DISCUSSIONS}

The ${ }^{13} \mathrm{C}$-NMR spectrum of $8 \mathrm{CB}$ in smectic phase can be seen in Fig. 2. In the inset of this figure, the alkyl part of the spectrum at a temperature corresponding to the nematic mesophase is displayed. The aromatic part of the spectrum keeps the same features up to the nematic-isotropic transition temperature. The position of the methyl carbon, $\mathrm{C}(\omega)$, in the isotropic phase is at $14.1 \mathrm{ppm}$, and it does not vary at the working temperatures due to its high mobility. Due to this fact, it has been taken as reference. The aromatic part of the carbon spectrum for $8 \mathrm{CB}$ is similar to those of other members of the $n \mathrm{CB}$ series, so we consider the updated assignments reported for $5 \mathrm{CB}$ in previous works. ${ }^{16} \mathrm{~A}$ detailed temperature dependence of the ${ }^{13} \mathrm{C}$ chemical shifts in $8 \mathrm{CB}$ and the complete assignments have been reported previously. ${ }^{21}$ The assignments for the alkyl carbons are supported by the segmental order parameters obtained from experimental $\mathrm{CP}$ frequencies (see below) and deuterium NMR experiments. ${ }^{22,23}$
In order to analyze the experimental data, it is necessary to correlate the geometry and symmetry of the molecular interactions with the cases presented in the theoretical section, associated with different values of $\kappa$, Eq. (8). For each methylene carbon in the aliphatic chain, the geometry of the molecule and the rapid rotations around the $\mathrm{C}-\mathrm{C}$ bonds, which lead to the trans-gauche isomerizations, allow us to take a single averaged value for both heteronuclear couplings-i.e., $b_{1} \approx b_{2}$. Then, for carbons $\mathrm{C}(\alpha)$ to $\mathrm{C}(\eta)$ in the aliphatic chain the relation of signs of the hetereonuclear couplings corresponds to the case where $\kappa=3$. It is also possible to see from simulations done in $5 \mathrm{CB}$ and from geometrical considerations that the homonuclear dipolar interaction $d$ between protons belonging to the same methylene cannot be neglected. ${ }^{24}$ For each nonquaternary carbon in the phenyls rings $\mathrm{C}\left(3^{\prime}\right), \mathrm{C}\left(2^{\prime}\right), \mathrm{C}(2)$, and $\mathrm{C}(3)$, we have one directly bonded proton interacting with a dipolar coupling $b_{1}$; however, a careful analysis indicates that neither the ${ }^{1} \mathrm{H}-{ }^{1} \mathrm{H}$ interaction $d$ nor the coupling between the ${ }^{13} \mathrm{C}$ and the nearest nonbonded ${ }^{1} \mathrm{H}, b_{2}$, can be neglected. Considering the rigidity of the phenyl ring and the orientation of each internuclear vector with respect to the external field, we see that $b_{1} \approx-b_{2}$. Then, each nonquaternary aromatic carbon is part of a three-spin system, where both heteronuclear couplings can be considered having an averaged magnitude $b=\sqrt{\left[b_{1}{ }^{2}+b_{2}{ }^{2}\right] / 2}$ and different relative signs, leading to $\kappa=1$.

Let us consider the experimental results corresponding to the smectic phase. In this case, typical oscillations and relaxation of the ${ }^{13} \mathrm{C}$ polarization versus contact time $t_{c}$ are shown in Fig. 3. We remark that neither the quaternary carbons nor $\mathrm{C}(\omega)$ show oscillations in the $\mathrm{CP}$ experiment. In the last case, the reason is the high mobility of the methyl group that averages to zero the effective carbon-proton interaction. In all these experiments, the ${ }^{13} \mathrm{C}$ polarization has essentially reached its asymptotic value at $5 \mathrm{~ms}$. The absence of decay in the time regime analyzed allows us to neglect spin-lattice relaxation in the rotating frame $\left(T_{1 \rho}\right)$. We also note that the $\mathrm{CP}$ frequencies corresponding to methylene groups are higher than those of the aromatic cores. This is due to a particularly unfavorable angle $\left(\sim 60^{\circ}\right)$ between the internuclear carbon-proton vector and the external magnetic field in the case of the phenyl rings. Besides, the contribution of the homonuclear coupling to the frequency is much smaller when $\kappa=1$. In each dynamical curve shown in Fig. 3 , the ${ }^{13} \mathrm{C}$ polarization at the first maximum is lower than its asymptotic value. As explained in the theoretical section, this fact indicates that the homonuclear coupling is not negligible and allows its evaluation.

We have fitted the experimental $\mathrm{CP}$ data to the equations derived for the isotropic [Eq. (15)] and anisotropic [Eq. (19)] models presented in the theoretical section. In the last case, we have distinguished a purely dipolar anisotropy ( $\alpha=2$ and $R_{\perp}=R_{\|}$) from the most general case. For the smectic phase, it is seen in Fig. 3 that the isotropic model departs from the experimental points after approximately the second maximum, fitting very poorly the asymptotic behavior. The dipolar model constitutes an improvement over the isotropic one, without adding extra free parameters. However, a much bet- 


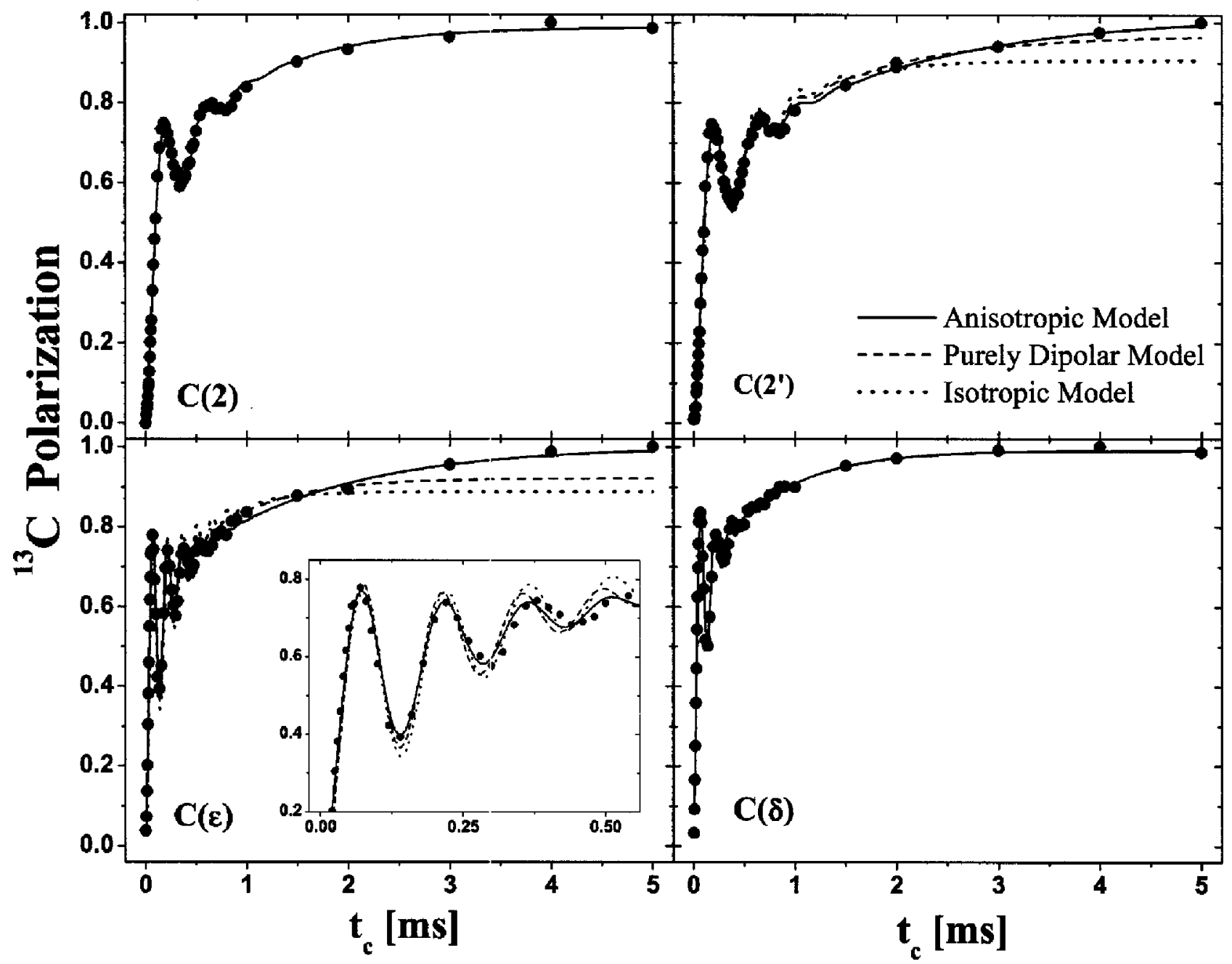

FIG. $3 .{ }^{13} \mathrm{C}$ polarization as a function of contact time $t_{c}$ for aromatic and aliphatic carbons in a standard CP experiment at $300 \mathrm{~K}$ (smectic phase). Fittings of the experimental data to the anisotropic, isotropic, and purely dipolar models described in the text.

ter fitting is obtained using the anisotropic model which follows very closely the first oscillations of the magnetization and it is the best in the asymptotic regime.

In the case of the standard $\mathrm{CP}$ experiments performed in the nematic phase up to $2 \mathrm{~ms}$ (not shown in the figure), the

TABLE I. Effective heteronuclear and homonuclear dipolar couplings $b$ and $d$ obtained by fitting the data of the standard CP experiment performed at $300 \mathrm{~K}$ (smectic phase) to the anisotropic model. The signal corresponding to $\mathrm{C}(\alpha)$ does not appear in the smectic spectra. The errors have been assigned considering Eqs. (21) for $b$ and $d$ in terms of the fitted parameters.

\begin{tabular}{ccc}
\hline \hline Carbons & $\begin{array}{c}b / 2 \pi \\
(\mathrm{kHz})\end{array}$ & $\begin{array}{c}d / 2 \pi \\
(\mathrm{kHz})\end{array}$ \\
\hline $\mathrm{C}\left(3^{\prime}\right)$ & $1.30 \pm 0.03$ & $4.9 \pm 0.3$ \\
$\mathrm{C}\left(2^{\prime}\right)$ & $1.49 \pm 0.04$ & $4.8 \pm 0.3$ \\
$\mathrm{C}(2)$ & $1.58 \pm 0.06$ & $4.9 \pm 0.3$ \\
$\mathrm{C}(3)$ & $1.45 \pm 0.04$ & $5.3 \pm 0.4$ \\
$\mathrm{C}(\beta)$ & $4.6 \pm 0.1$ & $4.5 \pm 0.6$ \\
$\mathrm{C}(\gamma)$ & $4.8 \pm 0.1$ & $5.9 \pm 0.7$ \\
$\mathrm{C}(\delta)$ & $4.7 \pm 0.3$ & $2.9 \pm 0.3$ \\
$\mathrm{C}(\varepsilon)$ & $4.3 \pm 0.2$ & $4.0 \pm 0.5$ \\
$\mathrm{C}(s)$ & $3.1 \pm 0.1$ & $3.7 \pm 0.4$ \\
$\mathrm{C}(\eta)$ & $3.2 \pm 0.1$ & $2.2 \pm 0.3$ \\
\hline \hline
\end{tabular}

behavior is already well fitted with the purely dipolar model, not showing any appreciable improvement by the use of the completely anisotropic model.

Taking into account the fitting parameters corresponding to each experiment, we can obtain the effective dipolar cou-

TABLE II. Effective heteronuclear and homonuclear dipolar couplings $b$ and $d$ for the aliphatic carbons obtained from the experiments performed at $311.5 \mathrm{~K}$ (nematic phase). In the standard $\mathrm{CP}$ experiment, the values are obtained by fitting the data to the purely dipolar model (see text). In the LG $\mathrm{CP}$, the values of $b$ are obtained directly from Eq. (22) while the values of $d$ requires a combination of the standard $\mathrm{CP}$ with the LG-CP frequencies [Eq. (25)]. In this mesophase the signal of $\mathrm{C}(\beta)$ merges to the signal of $\mathrm{C}(\delta)$, so the same value of coupling has been assigned to both carbons.

\begin{tabular}{ccccc}
\hline \hline & $\begin{array}{c}\text { Standard CP } \\
b / 2 \pi \\
(\mathrm{kHz})\end{array}$ & $\begin{array}{c}\text { LG-CP } \\
b / 2 \pi \\
(\mathrm{kHz})\end{array}$ & $\begin{array}{c}\text { Standard CP } \\
d / 2 \pi \\
(\mathrm{kHz})\end{array}$ & $\begin{array}{c}\text { LG-CP } \\
d / 2 \pi \\
(\mathrm{kHz})\end{array}$ \\
\hline $\mathrm{C}(\alpha)$ & $4.0 \pm 0.2$ & $4.18 \pm 0.04$ & $2.7 \pm 0.5$ & $2.9 \pm 0.6$ \\
$\mathrm{C}(\beta, \delta)$ & $2.7 \pm 0.1$ & $2.95 \pm 0.03$ & $3.5 \pm 0.6$ & $2.8 \pm 0.6$ \\
$\mathrm{C}(\gamma)$ & $3.0 \pm 0.2$ & $3.34 \pm 0.03$ & $3.5 \pm 0.7$ & $2.5 \pm 0.5$ \\
$\mathrm{C}(\varepsilon)$ & $2.8 \pm 0.1$ & $2.95 \pm 0.03$ & $3.1 \pm 0.5$ & $2.8 \pm 0.6$ \\
$\mathrm{C}(\mathrm{s})$ & $2.5 \pm 0.1$ & $2.29 \pm 0.03$ & $2.4 \pm 0.5$ & $2.8 \pm 0.5$ \\
$\mathrm{C}(\eta)$ & $2.4 \pm 0.1$ & $2.17 \pm 0.03$ & $1.5 \pm 0.3$ & $2.3 \pm 0.4$ \\
\hline \hline
\end{tabular}




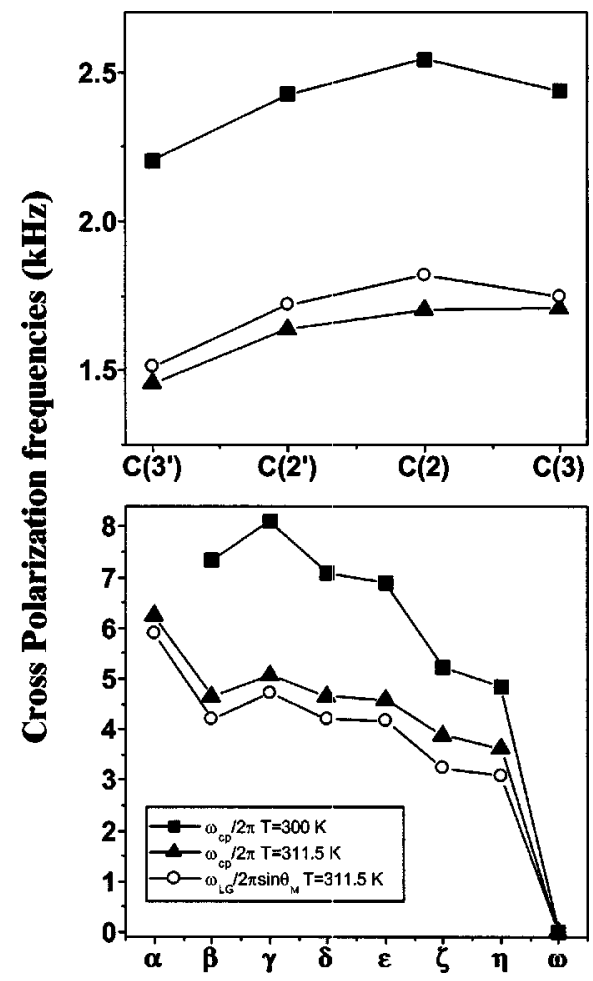

FIG. 4. Cross-polarization frequencies obtained from the LG and standard $\mathrm{CP}$ experiments for all nonquaternary carbons in the molecule. Note that the signal corresponding to $\mathrm{C}(\alpha)$ does not appear at $300 \mathrm{~K}$; also note that the signal of $\mathrm{C}(\beta)$ merges with that of $\mathrm{C}(\delta)$ at $311.5 \mathrm{~K}$, so the same value of frequency has been assigned to both carbons.

plings and the relaxation constants for each carbon showing oscillations during CP. In Tables I and II, we show the heteronuclear and homonuclear dipolar couplings at different temperatures. The values of the heteronuclear couplings are comparable with those obtained by Fung et al. ${ }^{25}$ where a different experimental technique was applied.

It is important to emphasize here that the frequency of the oscillations $\omega_{c p}$ is a quite independent and robust parameter, leading to values which vary less than $4 \%$ using different models. This allows one to determine the heteronuclear coupling $b$ with small error. The homonuclear coupling, however, is much more sensitive to the relation between the oscillatory and asymptotic regimes and so more dependent on the model and the extent of the experimental data. To clearly see this fact, we notice that the frequency and attenuation factor $f$ given in Eq. (7) are the fitting parameters that yield $b$ and $d$ regardless of the model. The dependencies between these parameters and the couplings are

$$
\begin{aligned}
& b / 2 \pi=(1 / \sqrt{2})\left(\omega_{c p} / 2 \pi\right) \sqrt{f}, \\
& d / 2 \pi=(4 / \kappa)\left(\omega_{c p} / 2 \pi\right) \sqrt{(1-f) .}
\end{aligned}
$$

In the cases studied here, $f$ is always bigger than $1 / 2$. Then, the error in $f$ (approximately $2 \%$ and $5 \%$ for the experiments in the smectic and nematic phases, respectively) affects $b$ less than $d$. Taking into account that the CP frequency $\omega_{c p}$ is the best parameter, fitted with an error below $2 \%$, we can assign the error of the other parameters of interest. Thus, the coupling $b$ is obtained with a relative error between $3 \%$ and $5 \%$ while the relative error of $d$ reaches in some cases a value of about $20 \%$.

Figure 4 displays the trend of the $\mathrm{CP}$ frequency as a function of ${ }^{13} \mathrm{C}$ position, obtained from the different experiments. In particular, for the aliphatic carbons, the expected zigzag pattern is observed..$^{22,23}$ For the aromatic part of the molecule we have $\omega_{c p}\left(3^{\prime}\right) \lesssim \omega_{c p}\left(2^{\prime}\right) \approx \omega_{c p}(2) \approx \omega_{c p}(3)$. This is expected because $\mathrm{C}\left(3^{\prime}\right)$, being the closest to the cyano group, forms a bigger $\mathrm{C}-\mathrm{C}-\mathrm{H}$ angle, giving rise to a smaller dipolar interaction. ${ }^{26}$ On the other hand, when increasing the temperature-i.e., going from smectic to nematic - a further averaging of the dipolar interactions occurs, manifested in the decrease of the CP frequencies. For comparison, Fig. 4 shows the results of the $\mathrm{CP}$ experiment at the Lee-Goldburg condition, performed in the nematic phase at the same temperature. In the LG experiment the $\mathrm{CP}$ frequency $\left(\omega_{L G}\right)$ is only related to the heteronuclear coupling because the homonuclear contribution has been quenched. Under this condition,

$$
\omega_{L G}=\sqrt{2 b^{2}\left(\sin \theta_{M}\right)^{2}},
$$

where $\theta_{M}=54.7^{\circ}$ is the magic angle. This angular factor comes from the projection of the rf field into the direction of the effective field for protons irradiated off resonance at the LG condition. ${ }^{27}$ Then, considering Eq. (7), we expect the relation

$$
\omega_{c p}>\omega_{L G} / \sin \theta_{M} .
$$

As shown in Fig. 4, this relation is not satisfied for aromatic carbons. Considering that $\mathrm{C}(\gamma)$ is exactly on resonance and that the $\mathrm{HH}$ condition was optimized for that carbon, the disagreement can be attributed to a non-negligible mismatch of the Hartmann-Hahn condition for carbons in the aromatic part of the spectrum. Taking into account a mismatch $\Delta$ for the aromatic carbons, the CP frequency for the LG experiment becomes

$$
\omega_{L G}=\sqrt{2 b^{2}\left(\sin \theta_{M}\right)^{2}+\Delta^{2}} .
$$

In the standard $\mathrm{CP}$ experiment, two frequencies appear, where the observable one is

$$
\omega_{c p}=\left(\omega_{c p}^{+}+\omega_{c p}^{-}\right) / 2,
$$

with

$$
\omega_{c p}^{ \pm}=\sqrt{\frac{(d \pm 4 \Delta)^{2}}{16}+2 b^{2}} .
$$

The other modulating frequency is too low to be observed in the presence of relaxation. Although it is difficult to quantify the exact magnitude of $\Delta$, we can see that the effect of the mismatch is greater for $\omega_{L G}$ than for $\omega_{c p}$, where there is a partial compensation between the two contributing frequencies $\omega_{c p}^{ \pm}$. This explains why $\omega_{L G} \gtrsim \omega_{c p}$ for the carbons in the aromatic part, in contrast with Eq. (23).

For carbons irradiated on resonance (aliphatic part), the values of the fitted parameters $b$ and $d$ obtained from standard CP experiments can be compared with the parameters obtained from the LG CP. In the latter case, the parameter of interest is $\omega_{L G}$. Then, $b$ is obtained in a direct way from 


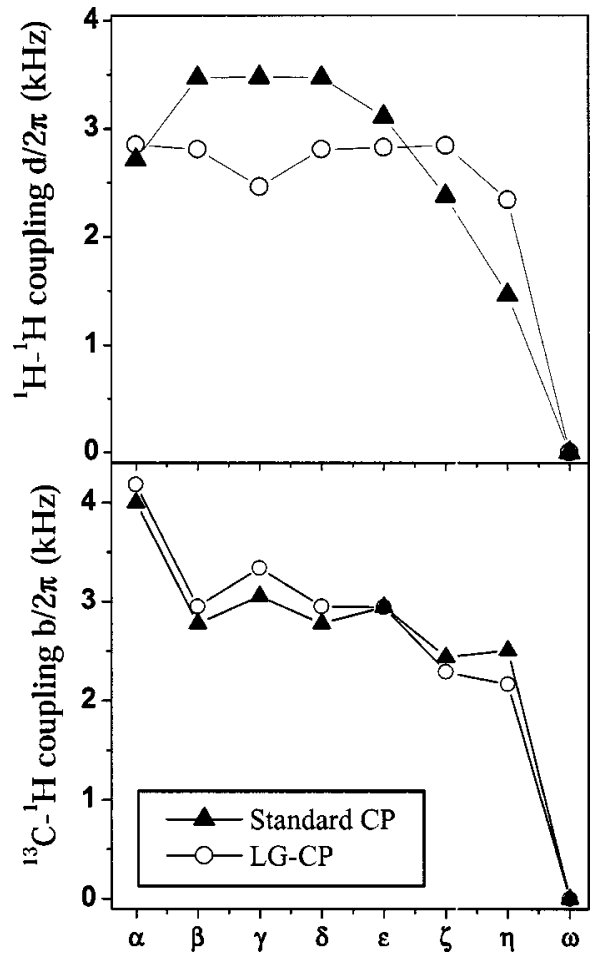

FIG. 5. Effective homonuclear $(d / 2 \pi)$ and heteronuclear $(b / 2 \pi)$ dipolar couplings for carbons in the alkyl chain obtained from the LG and standard $\mathrm{CP}$ experiments performed at $311.5 \mathrm{~K}$ (nematic phase).

expression (22) and $d$ can be obtained comparing $\omega_{L G}$ with the corresponding value of $\omega_{c p}$ at the same temperature. Using both experiments, $d$ is calculated from

$$
d=\frac{4}{\kappa} \sqrt{\omega_{c p}^{2}-\frac{\omega_{L G}^{2}}{\left(\sin \theta_{M}\right)^{2}}} .
$$

The values of $d$ calculated in this way with $\kappa=1$ (aliphatic carbons) can be compared with those obtained by fitting Eq. (19) to the standard CP data-i.e., coming from a single experiment.

Table II displays the values of the homonuclear and heteronuclear couplings for the aliphatic carbons obtained from the standard CP experiment and combining this with the LG $\mathrm{CP}$ performed at the same temperature. Figure 5 allows for the comparison of the ${ }^{13} \mathrm{C}-{ }^{1} \mathrm{H}$ and ${ }^{1} \mathrm{H}-{ }^{1} \mathrm{H}$ couplings ob-

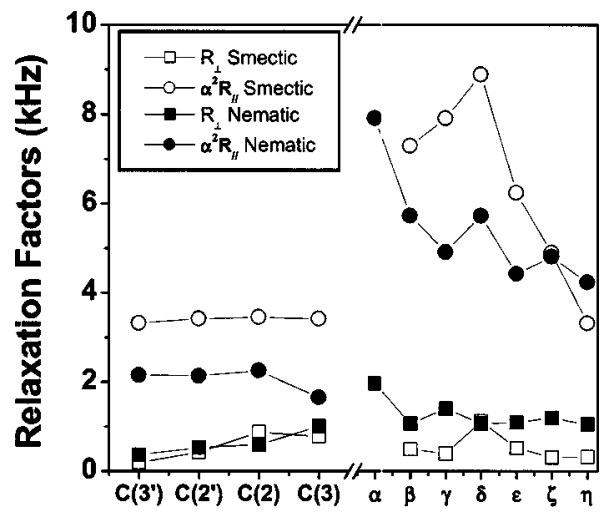

FIG. 6. Relaxation factors $\alpha^{2} R_{\|}$and $R_{\perp}$ obtained by fitting the standard CP data to the anisotropic model [Eq. (19)]. tained from the two methods. As expected, excellent agreement can be observed for the values of $b$. The novel methodology to estimate $d$ values yields good results within an error of around $20 \%$, which could be easily improved by taking data at longer times. Although there are few experimental measurements of these homonuclear couplings, those obtained directly from the ${ }^{1} \mathrm{H}$ spectra are in good agreement with the values obtained here.

With regard to the relaxation of the system in the $\mathrm{CP}$ experiments, we can observe an anisotropic behavior, quantified by the ratios $\alpha^{2} R_{\|} / R_{\perp}>1$, for both phases (see Fig. 6). Besides, we note that there is an important difference between the behaviors of the nematic and smectic phases. In the nematic phase, the anisotropy can be explained with a purely dipolar model. The average anisotropy factor for all carbons in the molecule is $(4 \pm 1)$. In contrast, in the smectic phase the factor $\alpha^{2} R_{\|} / R_{\perp}>4$ reveals a highly anisotropic behavior for most of the carbons. This can be appreciated in Fig. 3, where the amplitude of the first maximum is higher than the following ones, especially for the aliphatic carbons. This fact is not observed in nematics, giving support to the purely dipolar anisotropy. Different factors can produce this high anisotropy. One could be $H_{1}$ inhomogeneity; another could be an actual system size larger than $I_{2} S$. Although both factors would effectively increase the anisotropy, the effect should be comparable in both phases. Moreover, we have not observed such anisotropy ratios in solid molecular crystals, under the same $H_{1}$ inhomogeneity. Alternatively, the large anisotropy observed in the smectic phase could originate from the lack of fast fluctuations in this more rigid phase, which would prevent the application of the extreme narrowing approximation. If we depart from this approximation and assume that the spectral densities $J(0)$ and $J(\Sigma / 2)$, although different between them, are approximately constant in an interval of width $2 \omega_{c p}$, our calculations indicate that $R_{\|}$is proportional to $J(0)$, while $R_{\perp} \propto J(\Sigma / 2)$. Since usually $J(0)>J(\Sigma / 2)$, this could explain the higher anisotropy in the smectic phase, where motion is more constrained than in the nematic phase.

\section{CONCLUSIONS}

In this work, we presented ${ }^{13} \mathrm{C}-{ }^{1} \mathrm{H}$ cross polarization experiments which, complemented with detailed spin dynamics calculations, allowed us to obtain separately the homonuclear and heteronuclear dipolar couplings in $I_{2} \mathrm{~S}$ systems. The reliability of the obtained values was tested with a direct measurement of the heteronuclear couplings using CP under Lee-Goldburg conditions. Comparing both experiments we can conclude that LG CP is better for directly determining ${ }^{13} \mathrm{C}-{ }^{1} \mathrm{H}$ couplings without measuring for long contact times. However, the standard CP allows one to obtain homonuclear couplings in addition to the heteronuclear couplings. This experiment is also useful to get further information of the system such as the sources of relaxation.

On the other hand, from the theoretical analysis of the $I_{2} S$ dynamics, we recognized two different time scales for the decoherence behavior given by $R_{\|}$and $R_{\perp}$. Moreover, the CP data showed that the rate of attenuation of the oscillations is much faster than the rate of polarization transfer 
from the bath. This anisotropy could be explained in the nematic phase by assuming a purely dipolar interaction between the three-spin system and the bath within the extreme narrowing approximation. In the smectic phase, however, the anisotropy is much more pronounced and it seems that the approximation of rapid fluctuations in the bath is not appropriate. A slow-motion regime, controlled by the spin dynamics of the bath, would lead to a better agreement with the experimental observations without resorting to other mechanisms which operate in both phases.

\section{ACKNOWLEDGMENTS}

We acknowledge the ECOS-SUD Argentine-France cooperation program. Financial support was received from Fundación Antorchas, CONICET, FoNCyT, and SeCyTUNC. P.R.L. and H.M.P. are members of the Research Career and A.K.C. a Postdoctoral Fellow of Fundación Antorchas. G.A.A. is a Doctoral Fellow of CONICET.

${ }^{1}$ G. Salis, Y. Kato, K. Ensslin, D.C. Driscoll, A.C. Gossard, and D.D. Awschalom, Nature (London) 414, 619 (2001); A.V. Khaetskii, D. Loss, and L. Glazman, Phys. Rev. Lett. 88, 186802 (2002).

${ }^{2}$ N.A. Gershenfeld and I.L. Chuang, Science 275, 350 (1997); D.G. Cory, M.D. Price, and T.F. Havel, Phys. Rev. D 120, 82 (1998); C.H. Bennett and D.P. Di Vincenzo, Nature (London) 404, 247 (2002).

${ }^{3}$ W.K. Rhim, A. Pines, and J.S. Waugh, Phys. Rev. Lett. 25, 218 (1970).

${ }^{4}$ S. Zhang, B.H. Meier, and R.R. Ernst, Phys. Rev. Lett. 69, 2149 (1992).

${ }^{5}$ M. Ernst, B.H. Meier, M. Tomasselli, and A. Pines, J. Chem. Phys. 108, 9611 (1998); Mol. Phys. 95, 849 (1998).

${ }^{6}$ P.R. Levstein, G. Usaj, and H.M. Pastawski, J. Chem. Phys. 108, 2718 (1998); G. Usaj, H.M. Pastawski, and P.R. Levstein, Mol. Phys. 95, 1229 (1998).

${ }^{7}$ H.M. Pastawski, P.R. Levstein, and G. Usaj, Phys. Rev. Lett. 75, 4310 (1995); H.M. Pastawski, G. Usaj, and P.R. Levstein, Chem. Phys. Lett. 261, 329 (1996).
${ }^{8}$ Z.L. Mádi, B. Brutscher, T. Schulte-Herbrüggen, R. Brüschweiler, and R. Ernst, Chem. Phys. Lett. 268, 300 (1997).

${ }^{9}$ J.S. Waugh, Mol. Phys. 95, 731 (1998).

${ }^{10}$ W.H. Zurek, Rev. Mod. Phys. 75, 715 (2003).

${ }^{11}$ D. Sakellariou, P. Hodgkinson, and L. Emsley, Chem. Phys. Lett. 293, 110 (1998).

${ }^{12} \mathrm{~K}$. Schmidt-Rohr and H.W. Spiess, Multidimensional Solid State NMR and Polymers (Academic Press, London, 1996).

${ }^{13}$ L. Müller, A. Kumar, T. Baumann, and R.R. Ernst, Phys. Rev. Lett. 32, 1402 (1974).

${ }^{14}$ R. Pratima and K.V. Ramanathan, J. Magn. Reson. Ser A 118, 7 (1996).

${ }^{15}$ J. Hirschinger and M. Hervé, Solid State Nucl. Magn. Reson. 3, 121 (1994).

${ }^{16}$ B.M. Fung, A.K. Khitrin, and K. Ermolaev, J. Magn. Reson., Ser. A 142, 97 (2000).

${ }^{17}$ A. Abragam, The Principles of Nuclear Magnetism (Clarendon Press, Oxford, 1961).

${ }^{18} \mathrm{~K}$. Blum, Density Matrix Theory and Applications (Plenum Press, New York, 1981).

${ }^{19}$ R.R. Ernst, G. Bodenhausen, and A. Wokaun, Principles of Nuclear Magnetic Resonance in One and Two Dimensions (Oxford University Press, Oxford, 1987).

${ }^{20}$ P. Reinheimer, J. Hirschinger, P. Gilard, and N. Goetz, Magn. Reson. Chem. 35, 757 (1997).

${ }^{21}$ A.K. Chattah, F.M. Cucchietti, M. Hologne, J. Raya, and P.R. Levstein, Magn. Reson. Chem. 40, 772 (2002).

${ }^{22}$ R.Y. Dong, Nuclear Magnetic Resonance of Liquid Crystals (Springer, New York, 1997).

${ }^{23}$ C.J.R. Counsell, J.W. Emsley, N.J. Heaton, and G.R. Luckurst, Mol. Phys. 54, 847 (1985).

${ }^{24}$ B. Stevensson, A.V. Komolkin, D. Sandstrom, and A. Malianik, J. Chem. Phys. 114, 2332 (2001).

${ }^{25}$ B.M. Fung, C. Poon, M. Gangoda, E.L. Enwall, T.A.D. Diep, and C.V. Bui, Mol. Cryst. Liq. Cryst. 141, 267 (1986).

${ }^{26}$ S. Caldarelli, M. Hong, L. Emsley, and A. Pines, J. Phys. Chem. 100, 18696 (1996).

${ }^{27}$ C.P. Slichter, Principles of Magnetic Resonance (Springer, New York, 1992). 